\title{
Carrier Dynamics and Absorption Properties of Gold-Hyperdoped Germanium: Insight Into Tailoring Defect Energetics
}

\author{
Sashini Senali Dissanayake,,${ }^{1,}$ Naheed Ferdous, ${ }^{2}$ Hemi H. Gandhi, ${ }^{3}$ David Pastor, ${ }^{3,4}$ Tuan T. Tran, ${ }^{5}$ \\ Jim S. Williams, ${ }^{5}$ Michael J. Aziz $\odot,{ }^{3}$ Eric Mazur, ${ }^{3}$ Elif Ertekin, ${ }^{2}$ and Meng-Ju Sher ${ }^{1, \dagger}$ \\ ${ }^{1}$ Department of Physics, Wesleyan University, Middletown, Connecticut 06459, USA \\ ${ }^{2}$ Department of Mechanical Science \& Engineering, University of Illinois at Urbana-Champaign, Illinois 61820, \\ USA \\ ${ }^{3}$ John A. Paulson School of Engineering and Applied Sciences, Harvard University, Cambridge, Massachusetts \\ 02138, USA \\ ${ }^{4}$ Departamento de Estructura de la Materia, Física térmica y Electrónica, Facultad de Ciencias Físicas, \\ Universidad Complutense de Madrid, Madrid 28040, Spain \\ ${ }^{5}$ Research School of Physics, Australian National University, Australia
}

(Received 20 October 2020; revised 14 April 2021; accepted 8 June 2021; published 23 June 2021)

\begin{abstract}
Hyperdoping germanium with gold is a potential method to produce room-temperature shortwavelength-infrared radiation (SWIR; 1.4-3.0 $\mu \mathrm{m}$ ) photodetection. We investigate the charge carrier dynamics, light absorption, and structural properties of gold-hyperdoped germanium (Ge:Au) fabricated with varying ion implantation and nanosecond pulsed laser melting conditions. Time-resolved terahertz spectroscopy (TRTS) measurements show that Ge:Au carrier lifetime is significantly higher than that in previously studied hyperdoped silicon systems. Furthermore, we find that lattice composition, sub-bandgap optical absorption, and carrier dynamics depend greatly on hyperdoping conditions. We use density functional theory (DFT) to model dopant distribution, electronic band structure, and optical absorption. These simulations help explain experimentally observed differences in optical and optoelectronic behavior across different samples. DFT modeling reveals that substitutional dopant incorporation has the lowest formation energy and leads to deep energy levels. In contrast, interstitial or dopant-vacancy complex incorporation yields shallower energy levels that do not contribute to sub-band-gap light absorption and have a small effect on charge carrier lifetimes. These results suggest that it is promising to tailor dopant incorporation sites of Ge:Au for SWIR photodetection applications.
\end{abstract}

DOI: 10.1103/PhysRevApplied.15.064058

\section{INTRODUCTION}

Short-wavelength-infrared radiation (SWIR, $\lambda=1.4$ $3.0 \mu \mathrm{m}$ ) photodetection is important for numerous applications in medicine, industrial imaging, agriculture, telecommunications, and surveillance [1]. Conventional SWIR photodetectors are fabricated from narrow-band-gap III-V or II-VI semiconductors (e.g., $\operatorname{In}_{x} \mathrm{Ga}_{1-x} \mathrm{As}$, InAs, $\mathrm{Pb}_{1-x} \mathrm{Se}_{x}, \mathrm{Hg}_{1-x} \mathrm{Cd}_{x} \mathrm{Te}$ ) that are heterogeneously integrated with silicon-CMOS electronics. These approaches result in photodetectors that are expensive, toxic, or require low temperature to function [1-3]. The development of novel semiconductor materials that overcome these limitations could greatly enhance the applicability of SWIR photodetection. In this study we investigate Ge:Au as a material candidate for Ge-based photodetectors for entire SWIR.

\footnotetext{
*sdissanayake@wesleyan.edu

†msher@wesleyan.edu
}

It has recently been shown that $\mathrm{Ge:Au}$ is a potential material candidate for low-cost, silicon-compatible room-temperature SWIR photodetection [4]. Ge:Au is Ge supersaturated with high concentrations of $\mathrm{Au}$ in a singlecrystalline phase. Ge with a band-gap energy of $0.67 \mathrm{eV}$ (or $1.66 \mu \mathrm{m}$ ) does not absorb a large fraction of SWIR photons, but the absorption can be red-shifted through Audopant-mediated sub-band-gap absorption. Au dopants in Ge produce energy levels that lie near the center of the band gap [5] resulting in a low rate of thermal ionization $[6,7]$ and, hence, room-temperature SWIR photodetection is possible. Gandhi et al. show that Ge:Au exhibits subband-gap room-temperature optoelectronic responses, and the sub-band-gap absorption coefficient is comparable to those of commercial SWIR III-V and II-VI materials [8].

In this work, we fabricate Ge:Au by ion implantation followed by pulsed laser melting (PLM) and rapid solidification $[9,10]$. First, $\mathrm{Au}$ ions at high concentrations are introduced by ion implantation. The high dose of dopant ions implanted in Ge results in structural damage and, at 
high doses, an amorphous surface layer. A single laser pulse is used to melt the defective surface layer. The melt depth extends beyond the damaged layer and, upon cooling, the material resolidifies epitaxially from the substrate into a high-quality single crystal $[9,10]$. The rapid solidification process must be slow enough to ensure highquality epitaxial growth and, at the same time, it must be fast enough to trap $\mathrm{Au}$ at ultra-high concentrations [11]. The properties of hyperdoped materials depend on the parameters used in ion implantation and laser melting processes.

Previous studies on hyperdoped systems were mainly focused on Si [12-20]. One carrier recombination study in chalcogen-hyperdoped Si reveals a decrease in lifetimes with increasing dopant concentrations [18]. One ongoing study of Au-hyperdoped $\mathrm{Si}(\mathrm{Si}: \mathrm{Au})$ shows very short charge carrier lifetimes of the order of $10 \mathrm{ps}$ [21], but another study reports sub-band-gap photodetection out to $2200 \mathrm{~nm}$ at room temperature [10]. Density functional theory (DFT) calculations have previously been applied to Si:Au to investigate the defect formation energy, band structure, and absorption coefficients [19,20]. The DFT results show that the substitutional $\mathrm{Au}$ defects in Si are more energetically favorable than the interstitials [20]. Similar to previous Si studies, for hyperdoped Ge, structural and property studies and theoretical modeling of defect energetics are important for developing sub-bandgap optoelectronic devices.

In this work, we use a combination of experimental measurements and first principle DFT modeling to characterize and explain the origin of the unique structural and optoelectronic properties of Ge:Au. Specifically, we investigate the charge carrier dynamics, light absorption, and structural properties of $\mathrm{Ge}: \mathrm{Au}$ fabricated with varying ion-implantation and PLM-processing conditions. Time-resolved terahertz spectroscopy (TRTS) measurements show that Ge:Au carrier lifetime is significantly higher than that in previously studied hyperdoped silicon systems. We also find that optical and optoelectronic properties of Ge:Au critically depend on the Au lattice position, which can be controlled by specific fabrication conditions.

\section{METHODS}

\section{A. Sample fabrication and characterization}

In preparing hyperdoped $\mathrm{Ge}: \mathrm{Au}$ samples, $\mathrm{Au}$ ions are implanted at two doses $1 \times 10^{14}$ or $6 \times 10^{14} \mathrm{~cm}^{-2}$ using a $110-\mathrm{keV}$ ion implanter. The samples are tilted $7^{\circ}$ off the axis normal to avoid channeling and kept at liquid nitrogen temperature during the implants. Heavy-ion implantation is commonly known to induce severe morphological change, such as porosity, to Ge substrates. However, keeping the substrate at liquid nitrogen temperature can suppress the porosity up to the dose of $10^{16} \mathrm{~cm}^{-2}$ [22]. As a result, the Ge substrate is free of porosity yet amorphized after $\mathrm{Au}$ implantation. Following ion implantation, PLM is used to restore the crystallinity. For PLM, a single nanosecond laser pulse is used to melt the samples at two different laser fluences, 0.24 or $0.49 \mathrm{~J} / \mathrm{cm}^{2}$. The laser wavelength is $355 \mathrm{~nm}$ from the third harmonic of a Nd:YAG laser, and the pulse duration is 4-ns full width at half maximum (FWHM). Previous work showed that the resulting material is single crystalline [4]. The samples are prepared with combinations of the two ion doses and two laser fluences, namely: high dose-high fluence (HD_HF), high dose-low fluence (HD_LF), low dose-high fluence (LD_HF), and low dose-low fluence (LD_LF).

We use TRTS to perform noncontact photoconductivity decay measurements and study carrier recombination dynamics. An amplified Ti:sapphire femtosecond laser (with $800-\mathrm{nm}$ central wavelength and $2-\mathrm{mJ}$ pulse energy at $1-\mathrm{kHz}$ repetition rate) is used for the TRTS experiment. The laser output is split into three by beam splitters where one path generates terahertz pulses via the air-plasma terahertz generation method, one path is doubled to $400 \mathrm{~nm}$ with a $0.5-\mathrm{mm}$ beta-barium borate (BBO) crystal as an optical pump to excite the charge carriers in the sample, and the last path is used for terahertz detection using a 1-mm ZnTe for electro-optical sampling and balanced photodiodes $[23,24]$. The excited carrier density is $1.9 \times$ $10^{19} \mathrm{~cm}^{-3}$ at which we verified the photoconductivity decay dynamics to be independent of carrier density. After photoexcitation by the pump pulse, the excited charge carriers interact with the terahertz probe pulse and attenuate the terahertz signal. The time separation between the pump pulse (duration of $35 \mathrm{fs}$ ) and the probe pulse (duration of $1.2 \mathrm{ps}$ ) is controlled by a $300-\mathrm{mm}$ delay stage achieving a time window of $2 \mathrm{~ns}$. The time evolution of terahertz transmission allows us to measure transient photoconductivity decays and evaluate carrier lifetimes with picosecond time resolution.

Rutherford backscattering spectrometry (RBS) is employed to characterize the implanted Au profile, the amorphous layer thickness and the crystal properties of the as-implanted and the post-PLM samples. A monoenergetic $2 \mathrm{MeV} \mathrm{He}^{+}$ion beam backscattered from the samples is detected by a solid-state detector at an angle of $100^{\circ}$. For the as-implanted samples, only the channeling geometry, in which the ion beam is parallel to the [100] axis of the substrates, is employed. This allows us to study the $\mathrm{Au}$ distribution and the thickness of the amorphous layer. For the post-PLM samples, both random and channeling geometries are employed for the quantification of the $\mathrm{Au}$ redistribution, the crystal quality, and the substitutionalinstitutional fraction of the implanted species. Analysis of the RBS spectra is performed using the SIMNRA simulation program [25]. Considering most scattering events at $2 \mathrm{MeV}$ are single-scattering, the program can use analytical formulae to speed up the simulation. For the electronic stopping power, we chose the SRIM option because it is 
a semiempirical database relying on existing experimental data and provides more reliable stopping values.

The optical absorptance $(A)$ of each sample is determined by the measured transmittance $(T)$ and the reflectance $(R)$, and calculated using the relation $A=1-$ $T-R$. The transmittance and reflectance of each sample are measured using an ultraviolet-visible-near-infrared (UV-VIS-NIR) spectrophotometer equipped with an integrating sphere. The optical absorptance of the Ge substrate is also measured for comparison purposes.

\section{B. Computational methods}

We simulate Ge:Au using DFT [26,27] employing the Vienna $A b$ initio Simulation Package (VASP) $[28,29]$. The screened hybrid functional of Heyd, Scuseria, and Ernzerhof (HSE06) $[30,31]$ is implemented for the description of exchange and correlation. This is necessary because standard functionals such as local-density approximation (LDA) [27] and Perdew-Burke-Ernzerhof (PBE) [32,33] close the band gap in Ge. We use the standard parameters for exchange mixing and range separation with HSE06.

Projector augmented wave (PAW) pseudopotentials are used, and the orbitals are expanded in a plane wave basis set with kinetic energy cutoff of $460 \mathrm{eV}$. Our optimized lattice constant of bulk Ge, $5.65 \AA$, is similar to the experimental value of $5.658 \AA$ [34]. The HSE06-obtained indirect (direct) band gap, $0.81 \mathrm{eV}(0.90 \mathrm{eV})$ slightly overestimates the experimental band gap, $0.66 \mathrm{eV}$ [35] (0.80 $\mathrm{eV}$ [35]). For defect calculations, 128-atom supercells are used and the Brillouin zone is sampled with a MonkhorstPack $2 \times 2 \times 2 k$-point mesh. Total energies are converged within $0.01 \mathrm{eV}$ and atomic coordinates are relaxed until the forces on each atom are less than $0.01 \mathrm{eV} / \AA$.

We consider Au-related defects that are most likely to appear in Ge:Au. Although Au energetically prefers substitutional sites in $\mathrm{Ge}$ [36], both substitutionals $\mathrm{Au}_{\mathrm{Ge}}$ and tetrahedral interstitials $\mathrm{Au}_{i, \text { tetr }}$ are considered because the laser-implantation process may result in nonequilibrium defect distributions. In addition, there is evidence that ion implantation followed by PLM can leave behind a substantial, nonequilibrium population of vacancies that can trap or otherwise interact with the dopant atoms [19]. Therefore, we also consider complexes that may form between $\mathrm{Au}$ and vacancies. Amongst the large set of possible interactions, the most common is a single vacancy that traps a diffusing interstitial $\mathrm{Au}$ resulting in a divacancy-interstitial $\mathrm{Au}$ complex $\mathrm{Au}_{i}-2 \mathrm{~V}[37,38]$.

For $\mathrm{Au}_{\mathrm{Ge}}, \mathrm{Au}_{i, \text { tetr }}$, and $\mathrm{Au}_{i}-2 \mathrm{~V}$, defect formation energies are obtained by

$$
\triangle E_{D, q}=\left(E_{D, q}-E_{\mathrm{perf}}\right)-\sum_{i} n_{i} \mu_{i}+q\left(E_{v}+E_{F}\right),
$$

where $E_{D, q}$ is the DFT total energy of the defect incorporating supercell, $E_{\text {perf }}$ is the total energy of the supercell with no defect present, $n_{i}$ is the number of added $\left(n_{i}>0\right)$ or removed $\left(n_{i}<0\right)$ species $i(i=\mathrm{Ge}, \mathrm{Au})$ for generating the defect, and $\mu_{i}$ is the atomic chemical potential of the participating species. The chemical potentials $\mu_{\mathrm{Ge}}$ and $\mu_{\mathrm{Au}}$ reflect $\mathrm{Ge}$ and $\mathrm{Au}$ rich experimental conditions, and are obtained by assuming equilibrium with the elemental bulk phases of $\mathrm{Ge}$ and $\mathrm{Au}$, respectively. The electron chemical potential $E_{F}$ (Fermi level) is referenced to the DFT valence band maximum $E_{v}$.

The optical absorption coefficient $\alpha$ is obtained from the imaginary part of the dielectric function $\epsilon^{\mathrm{im}}(\omega)$ using linear response theory [39]. The real part of the dielectric tensor can then be obtained from the Kramers-Kronig relations [40]. As the calculated $\alpha$ considers only direct excitations, there may be discrepancies with experimental measurements which also include indirect phonon-assisted transitions.

\section{STRUCTURAL AND OPTOELECTRONIC PROPERTIES}

\section{A. Carrier decay dynamics and structural analysis}

Figure 1(a) shows photoconductivity decay dynamics for the four samples. The half-life of photoconductivity decays are $0.86,1.00,0.95$, and $0.18 \mathrm{~ns}$ for LD_HF, LD_LF, HD_HF, and HD_LF, respectively. The HD_HF sample shows similar decay dynamics as the two LD samples and, in contrast, HD_LF exhibits a significantly shorter lifetime. Figure 1(b) shows light absorption of each sample with wavelengths ranging from visible to SWIR. Both the substrate and LD_HF have near-zero absorptance in the SWIR region. Also in this region, the HD_LF sample shows the highest absorptance compared with the other samples.

The properties of Ge:Au make it more suitable for applications in advanced optoelectronic devices. Ge:Au exhibits long charge carrier lifetimes compared with other hyperdoped materials. Considering $\mathrm{Si}: \mathrm{Au}$, a system with demonstrated room-temperature photoresponse to wavelengths as long as $2200 \mathrm{~nm}$, has a dopant concentration of $10^{20}$ atoms $/ \mathrm{cm}^{3}$ and sub-band-gap absorption less than $2 \%$ [10]. The dopant concentration and sub-band-gap light absorption of Si:Au in Ref. [10] are comparable to the properties of $\mathrm{Ge}: \mathrm{Au}$ in this work. Si:Au, however, exhibits charge carrier half-life of 0.005 ns [21], while the Ge:Au in this work has half-life two orders magnitude longer. In addition, the lifetime of $\mathrm{Si}: \mathrm{Au}$ is a strong function of incorporated Au dose [42], with lifetime decreasing monotonically with increasing $\mathrm{Au}$ concentration. On the other hand, carrier lifetime of $\mathrm{Ge}: \mathrm{Au}$ samples in our study is additionally affected by different PLM conditions.

Structural differences in Au atom location between these samples are investigated by RBS to shed light on the differences in the charge carrier lifetimes. Figures 2(a) 

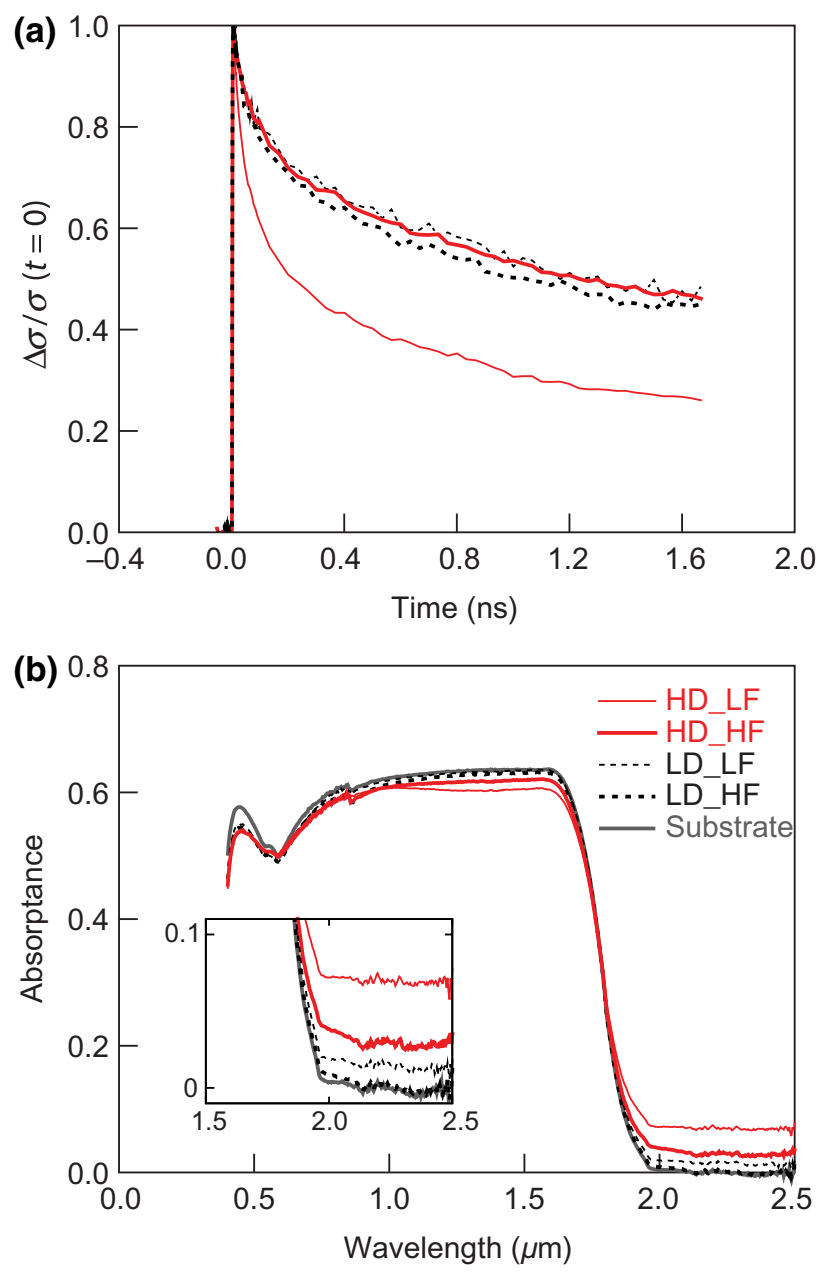

FIG. 1. (a) Photoconductivity of $\mathrm{Ge}: \mathrm{Au}$ as a function of time. HD_LF (thin red line), HD_HF (thick red line), LD_LF (thin black dashed line), and LD_HF (thick black dashed line) are different samples with different $\mathrm{Au}$ doses and PLM fluences. HD_LF has a significantly shorter lifetime than the rest of the samples. (b) Wavelength-dependent light absorptance of each sample. The Ge substrate is optically inactive in the sub-bandgap region whereas Ge:Au is optically active and HD_LF has the highest sub-band-gap light absorption.

and 2(b) show the total and substitutional Au concentrations as functions of depth, respectively. Figure $\mathrm{S} 1$ in the supplementary material shows the RBS spectra. The concentration is obtained up to $40 \mathrm{~nm}$, and beyond this depth the Au spectrum overlaps with the Ge spectrum, limiting our resolution. The structural data, however, is sufficient for us to understand the trends in light absorption and carrier recombination properties. In detail, when performing terahertz photoconductivity measurements, only the top $14 \mathrm{~nm}$ is excited due to the high absorption coefficient of $\mathrm{Ge}$ at the optical pump wavelength $(400 \mathrm{~nm})$. Comparing the two HD samples, the HD_HF Au distribution is highly segregated towards the surface in both total and

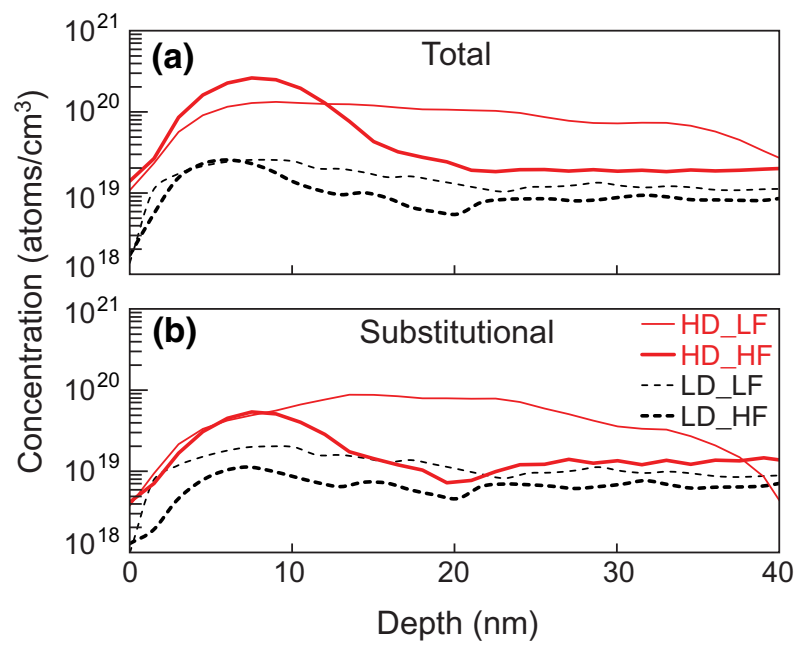

FIG. 2. Structural analysis of Au atom location from RBS measurements. The raw RBS data are shown in Fig. S1 in the supplementary material [41]. HD_LF sample has the highest substitutional concentration, and the HD_HF substitutional concentration is similar to LD samples.

substitutional concentration profiles. Considering the substitutional concentration profile, HD_LF has a higher substitutional concentration. Deeper into the sample, HD_HF has concentrations similar to LD samples.

Figure 3 summarizes the experimental results. Figure 3(a) represents the recombination rate as the inverse of half-life as well as the averaged sub-band-gap absorptance from 2.0 to $2.5 \mu \mathrm{m}$. Both the recombination rate and the absorptance exhibit similar trends across samples. According to

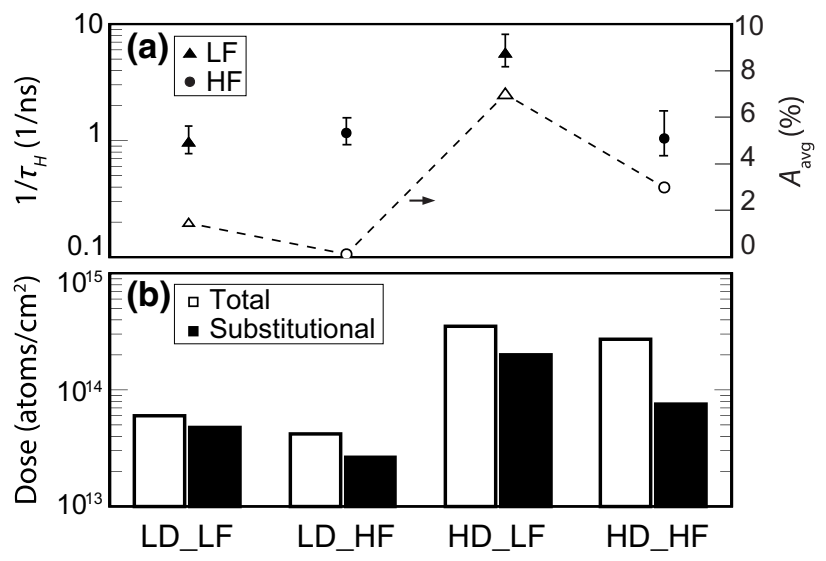

FIG. 3. (a) The inverse of half-lives (filled symbols) and subband-gap absorptance (unfilled symbols) show similar trends. Triangles and circles represent LF and HF, respectively. (b) Total and substitutional dose of $\mathrm{Au}$ in hyperdoped $\mathrm{Ge}$ samples from RBS analysis. The unfilled bars and filled bars represent the total and substitutional doses, respectively. HD_LF has the most substitutional $\mathrm{Au}$ incorporation, whereas substitutional $\mathrm{Au}$ incorporation in the HD_HF sample is similar to the two LD samples. 
the Shockley-Reed-Hall (SRH) recombination model for defect-assisted carrier trapping and recombination, carrier lifetime should decrease as dopant concentration increases [43]. The SRH model is based on statistics of charge carrier capturing processes by deep-level trap states which are, in this case, the Au dopants. Assuming a trap state has carrier capture cross section $\sigma$ and is not filled, the carrier capturing rate is $N_{t} V_{\mathrm{th}} \sigma$, where $N_{t}$ is the density of the traps and $V_{\text {th }}$ is the thermal velocity of electrons. Therefore, the recombination rate would increase with increasing dopant concentration. When the PLM fluence is low (filled triangle symbols), recombination rate indeed increases with $\mathrm{Au}$ dose. When the PLM fluence is high (filled circle symbols), on the other hand, the lifetimes of the two samples are comparable and do not exhibit trends predicted by the SRH model. To understand why the recombination rate for HD_HF is slower than expected, we analyze structural properties of the samples in detail.

Figure 3(b) shows the total and substitutional Au dose by integrating the concentrations-depth profile from 0 to $40 \mathrm{~nm}$. The total dose of the two HD samples is approximately one order magnitude higher than the total dose of the two LD samples. Both LD samples are highly substitutional, where the substitutional fraction is $80 \%$ and $65 \%$ for LF and HF, respectively. The substitutional fraction for HD_LF is $60 \%$ and HD_HF is lower at $30 \%$. In the RBS analysis shown in Figs. 2 and 3, we find that the amount of substitutional incorporation is strongly dependent on laser fluence. In particular, the HD_HF sample has a significant amount of Au dopant segregated toward the surface, resulting in a lower substitutional incorporation.

Figure 3(a) shows that the light absorption and carrier recombination rate are both highest in HD_LF, and both properties for HD_HF are similar to the LD samples. Even though the total dose of the HD_HF sample is one order magnitude larger than the LD samples, both the recombination rate and the light absorption properties are closer to the LD samples. Comparing the two HD samples, there is a significant difference in substitutional dose. In the HD_HF sample, the majority of the Au dopant resides in sites that are not substitutional, and the nonsubstitutional dopant has a small effect on charge carrier lifetime or absorption (Fig. S2 in the supplementary material [41]). To shed light on how the Au atom location site influences properties, we study defect energetics.

\section{B. Defect energetics}

Figures 4(a)-4(c) show the three types of defect sites considered in this work, $\mathrm{Au}_{\mathrm{Ge}}, \mathrm{Au}_{i, \text { tetr, }}$, and $\mathrm{Au}_{i}-2 \mathrm{~V}$. Figure 4(d) is the calculated formation energies. Formation energies of different defects indicate the relative probability of defect formation. The most probable defect is that with the smallest formation energy. Owing to the ultra-high concentrations, the hyperdoped samples, however, are not in an equilibrium state, and thus other high-energy defects can also form. The formation energies are $1.61 \mathrm{eV}$ for $\mathrm{Au}_{\mathrm{Ge}}, 3.25 \mathrm{eV}$ for $\mathrm{Au}_{i, \text { tetr }}$, and $2.80 \mathrm{eV}$ for $\mathrm{Au}_{i}-2 \mathrm{~V}$. Here $\mathrm{Au}_{\mathrm{Ge}}$ has the lowest $E_{D, q}$ and, thus, is the most favorable defect in Ge:Au system.

Although $\mathrm{Au}_{\mathrm{Ge}}$ has the lowest formation energy, the formation of the other defects remains possible. For example, despite its high energy, $\mathrm{Au}_{i \text {,etr }}$ may form via kickout or dissociative mechanisms, as believed to occur for $\mathrm{Si} A \mathrm{Au}$ upon thermal annealing [44]. In addition, $\mathrm{Au}_{i}-2 \mathrm{~V}$ can form when a substitutional $\mathrm{Au}$ and monovacancy are placed side by side and allowed to relax; the Au atom is attracted towards the vacancy resulting in the lattice distortion as shown in Fig. 4(c). In the final geometry, the Au atom sits in an interstitial site just between two silicon vacancies. Details of the geometries can be found in Ref. [20], where $\mathrm{Au}_{i^{-}}$ $2 \mathrm{~V}$ was shown to play a role in the thermal relaxation of Si:Au. A previous study on Si:Au showed that PLM introduces a high vacancy concentration [45]. If vacancies are also present in hyperdoped $\mathrm{Ge}$, then defect reactions between isolated vacancies and $\mathrm{Au}_{\mathrm{Ge}}$ can result in $\mathrm{Au}_{i^{-}}$ $2 \mathrm{~V}$. The binding energy between an $\mathrm{Au}_{\mathrm{Ge}}$ and a vacancy is determined here to be $1.74 \mathrm{eV}$.

It is possible to roughly estimate how different defects may affect carrier lifetimes based on their electronic band structure shown in Fig. 5. The defect-induced bands are shown in red in all cases. Due to computational resource
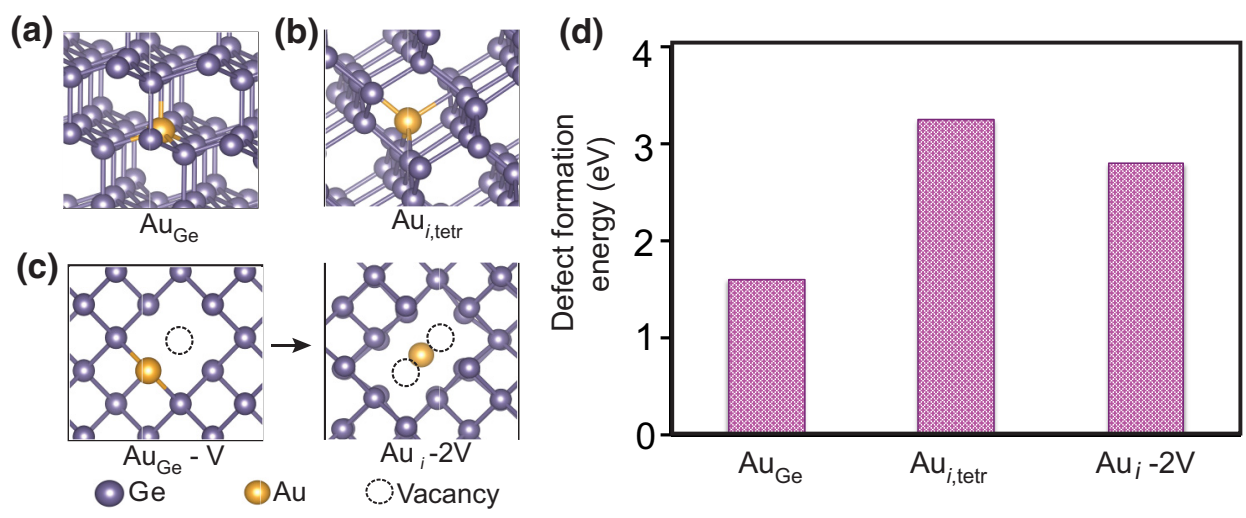

FIG. 4. (a) Isolated substitutional, $\mathrm{Au}_{\mathrm{Ge}}$, (b) tetrahedral interstitial, $\mathrm{Au}_{i, \text { tetr }}$, (c) gold-vacancy complex, $\mathrm{Au}_{i}-2 \mathrm{~V}$, and (d) the DFT deduced defect formation energies, $E_{D, q}$, of the substitutional $\mathrm{Au}_{\mathrm{Ge}}$, interstitial $\mathrm{Au}_{i, \text { tetr, }}$, and impurityvacancy complex $\mathrm{Au}_{i}-2 \mathrm{~V}$. 


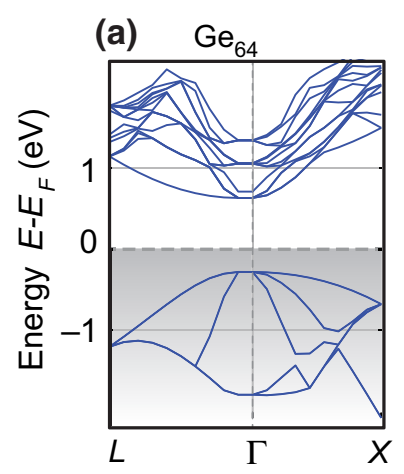

(b) $\mathrm{Au}_{\mathrm{Ge}}$

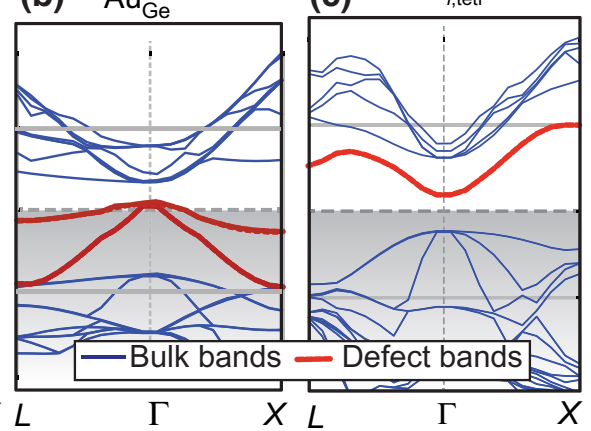

(d) $\mathrm{Au}_{i}-2 \mathrm{~V}$

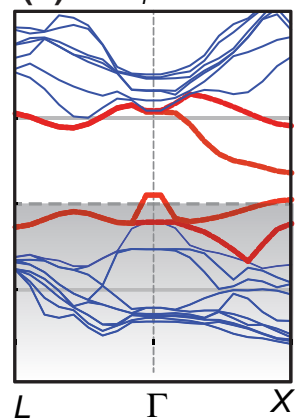

FIG. 5. (a) Band structure of pure $\mathrm{Ge},(\mathrm{b})$ localized trap states of $\mathrm{Au}_{\mathrm{Ge}}$, (c) isolated single energy state from $\mathrm{Au}_{i, \text { tetr }}$, and (d) delocalized bands coming from $\mathrm{Au}_{i}-2 \mathrm{~V}$. The results are shown for 0.78 at. \% concentration of Au. The Fermi level is shown by the dashed black horizontal line, below which all bands are filled. limitations defects are simulated at a fixed concentration (in 128-atom supercells) of 0.78 at. \% $\mathrm{Au}$, a factor of three higher than the experimentally observed HD_LF sample of 0.23 at. \%. This affects the dispersion in the defect levels shown in Fig. 5, but it is still possible to capture the main features of the physics from the qualitative nature (Fig. S3 in the supplementary material [41]). Details of the origin of bands (similar to Au related defects in $\mathrm{Si}$ ) can be found in Refs. $[20,46]$.

From Fig. 5(b) we see that $\mathrm{Au}_{\mathrm{Ge}}$ introduces three (two of which are degenerate) mid-gap defect bands. For the 128atom supercells, these bands appear to slightly merge with the valence bands, although this is likely the result of the high simulated concentrations. The Fermi level lies within the defect bands, meaning they are partially filled. $\mathrm{Au}_{\mathrm{Ge}}$ therefore may serve as a trap for photoexcited electrons in the conduction band or holes in the valence band.

Were it to be present, the isolated defect level of $\mathrm{Au}_{i, \text { tetr }}$ in Fig. 5(c) would suggest that it may act as a trap, particularly for electrons in the conduction band. These traps are taken as an indication of loss of carrier lifetime.

In Fig. 5(d), the electronic structure of $\mathrm{Au}_{i}-2 \mathrm{~V}$ shows defect levels away from mid-gap, offset towards both the conduction and valence bands. There are no mid-gap defect levels present. We expect that such a defect complex, were it to be present, would have a more benign effect on carrier lifetimes. Although this study provides no direct evidence of $\mathrm{Au}_{i}-2 \mathrm{~V}$ in particular, prior experimental evidence of an $\mathrm{Au}_{i}$-vacancy complex was found using $\mathrm{Au}$ radiotracer diffusion experiments [47]. We note that $\mathrm{Au}_{i^{-}}$ $2 \mathrm{~V}$ is one simple example of a defect complex that may form in $\mathrm{Ge}: \mathrm{Au}$, considered here only based on our prior experience with $\mathrm{Si}: \mathrm{Au}$. The experimentally realized systems are expected to show a richer phase space of defect complexes. However, this example shows that reduced substitutional fraction may result in complexes, some of which are more benign to transport than the substitutional defects.

Figure 6 shows the computed absorption spectra for all three defects. Note two main sources of discrepancy between the calculated absorption coefficient and experimentally measured values: the exclusion of indirect phonon-assisted transitions and the large defect concentrations used in the simulations. Our calculated results therefore provide a qualitative understanding.

As Fig. 6 shows, the substitutional $\mathrm{Au}_{\mathrm{Ge}}$ provides the largest sub-band-gap absorption, consistent with the partially filled, dispersive mid-gap states in the electronic structure. Thus, large incorporation of substitutional $\mathrm{Au}$ into our samples would correlate to large optical enhancement. In contrast, $\mathrm{Au}_{i \text {,etr }}$ shows the least sub-band-gap absorption, owing to the presence of only one mid-gap defect level. Compared with substitutional $\mathrm{Au}$, the optical response turns on at higher photon energies, because the mid-gap level is completely empty and allows transitions only from the valence band (VB) to the defect level itself. Meanwhile, $\mathrm{Au}_{i}-2 \mathrm{~V}$ shows sub-band-gap absorption in between that of $\mathrm{Au}_{\mathrm{Ge}}$ and $\mathrm{Au}_{i, \text { tetr }}$, arising from transitions between the defect bands near the VB and the conduction band $(\mathrm{CB})$. For these more delocalized defect bands, the optical transitions again turn on at higher photon energies than that of $\mathrm{Au}_{\mathrm{Ge}}$. The boost in absorption below band gap is due to the fact that simulated high concentration causes dispersed defect bands that yield increased defect-defect interaction. The important fact is that the $\mathrm{Au}_{i}-2 \mathrm{~V}$ defect bands are not intermediate bands, but are bulklike bands that will generate absorption near the band edges. For that reason, $\mathrm{Au}_{i}-2 \mathrm{~V}$ is not expected to yield sub-band-gap

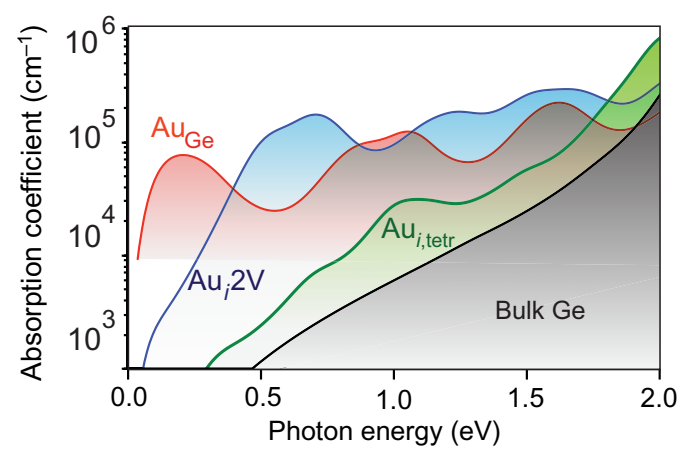

FIG. 6. DFT-HSE06-obtained absorption coefficients of $\mathrm{Au}_{\mathrm{Ge}}$, $\mathrm{Au}_{i, \text { tetr }}$, and $\mathrm{Au}_{i}-2 \mathrm{~V}$ comparing them with the absorption coefficient of bulk Ge. The HSE06 direct band gap is $0.88 \mathrm{eV}$. 
absorption and we should focus on the low-photon-energy region of Fig. 6 for qualitative comparison. Note that the HSE0 6 band gap of $\mathrm{Ge}$ is at $0.88 \mathrm{eV}$, and in Fig. 6, the subband-gap absorption shown for bulk Ge arises as an artifact of the Gaussian smearing used to numerically smooth the behavior. Owing to the band gap overestimate in DFT, the precise numerical values where the optical response turns on for each defect may not numerically match the corresponding experimental value. Even so, the trends described with respect to the relative optical activity of the different configurations are expected to hold.

\section{DISCUSSION}

Many observed properties of Ge:Au samples can be explained by the DFT simulation results. Most of the samples studied are highly substitutional (above 60\% Au substitutional site incorporation), which is consistent with DFT predicting that substitutional Au has the lowest formation energy.

Experimentally we also find correlations between substitutional fraction, lifetime, and absorption strength across samples. First, there is a clear correlation between substitutional dose and sub-band-gap light absorption across samples, where the absorption increases with increasing substitutional dose. Second, the half-life of the samples decreases with increasing substitutional dose. DFT simulations explain these findings by illuminating the different energy levels produced by $\mathrm{Au}$ dopants incorporated on different lattice sites. We find that substitutional $\mathrm{Au}$ in Ge produces deep energy levels, whereas interstitial and dopant-vacancy complexes yield shallower energy levels near band edges. Substitutional Au-dopants therefore reduce carrier lifetime and produce strong sub-band-gap light absorption, whereas nonsubstitutional dopants have a minimal effect on both carrier lifetime and sub-band-gap light absorption.

This study reveals that controlling defect incorporation site is critical in optimizing $\mathrm{Ge:Au}$ optoelectronic properties for SWIR photodetection. The hyperdoping process used is a highly nonequilibrium fabrication method, and PLM fluence affects dopant incorporation. With increasing PLM fluence, the resolidification speed is slower, and fewer $\mathrm{Au}$ dopants are incorporated. Simulations carried out in Ref. [4] show that both melt depth and melt duration increase with fluence. The maximum resolidification velocity decreases at high fluence, leading to less Au trapping (Fig. S4 in the supplementary material [41]). In our experiment, the HF samples show more segregation in the dopant profiles (Fig. 2) with HD_HF mostly segregated toward the surface, whereas the HD_LF sample shows a much more uniform concentration profile. As a result, the HD_HF sample has only $30 \%$ of the Au dopants in substitutional sites, lower than the HD_LF sample at $60 \%$, whereas both samples have comparable total Au dose. Our results show that optimizing PLM fluence is crucial for placing Au dopants in substitutional sites.

\section{CONCLUSIONS}

In summary we carry out experimental and computational analyses to characterize and explain the unique optoelectronic properties of Ge:Au for SWIR photodetection. Ge:Au fabricated using ion implantation followed by PLM yields high-quality materials with high substitutionality for a set of samples with varying Au doses and laser fluences. Sub-band-gap absorption is experimentally observed, and the measured charge carrier lifetime is significantly longer than hyperdoped Si. First-principles DFT is employed to find the reason behind high absorption and low carrier lifetime of the HD_LF sample. The formation of isolated intermediate bands for substitutional $\mathrm{Au}$ is suggested to give rise to high absorption coefficient while serving as the carrier recombination site. Our study suggests that performance of Ge:Au SWIR photodetectors can be optimized by tailoring the Au implantation and PLM hyperdoping processes to minimize carrier recombination and maximize sub-band-gap absorption.

\section{ACKNOWLEDGMENTS}

H.H.G. acknowledges support from the Department of Defense (DoD) under Grant Nos. DGE 0946799 through the National Defense Science and Engineering Graduate Fellowship (NDSEG) Program and the Directed Energy Processing Society Graduate Fellowship. D.P. acknowledges financial support from the MEC within Programa Nacional de movilidad de recursos humanos del Plan Nacional I+D+i 2008-2011 (EX-2010-0662), the program Ramón y Cajal (RYC-2014-16936) and from the Spanish ministry of Science, Innovation and Universities (MICINN) under contract no. TEC2017-84378-R. This work is part of the project MADRID-PV2 P-2018/EMT4308 funded by the Regional Government of Madrid with support from FEDER funds. This work was also supported by the US Air Force Office of Scientific Research (FA9550-14-1-0150). This work was performed in part at the Harvard Center for Nanoscale Systems (CNS), a member of the National Nanotechnology Infrastructure Network (NNIN), which is supported by the National Science Foundation under NSF award no. ECS-0335765.

\section{DATA AVAILABILITY}

The data that support the findings of this study are available from the corresponding author upon reasonable request. 
[1] Marc P. Hansen and Douglas S. Malchow, Overview of SWIR detectors, cameras, and applications, Thermosense XXX 6939, 94 (2008).

[2] T. E. Kazior, J. R. LaRoche, D. Lubyshev, J. M. Fastenau, W. K. Liu, M. Urteaga, W. Ha, J. Bergman, M. J. Choe, M. T. Bulsara, E. A. Fitzgerald, D. Smith, D. Clark, R. Thompson, C. Drazek, N. Daval, L. Benaissa, and E. Augendre, 2009 IEEE International Conference on Indium Phosphide Related Materials (2009), p. 100. ISSN 1092-8669.

[3] T. E. Kazior, Beyond CMOS: Heterogeneous integration of III-V devices, RF MEMS and other dissimilar materials/devices with Si CMOS to create intelligent microsystems, Philos. Trans. A: Math. Phys. Eng. Sci. 372, 20130105 (2014). ISSN 1364-503X (Print) 1364-503X (Linking).

[4] Hemi H. Gandhi, David Pastor, Tuan T. Tran, S. Kalchmair, L. A. Smilie, Jonathan P. Mailoa, Ruggero Milazzo, Enrico Napolitani, Marco Loncar, James S. Williams, Michael J. Aziz, and Eric Mazur, Gold-Hyperdoped Germanium with Room-Temperature Sub-Band-Gap Optoelectronic Response, Phys. Rev. Appl. 14, 064051 (2020).

[5] L. Johnson and H. Levinstein, Infrared properties of gold in germanium, Phys. Rev. 117, 1191 (1960).

[6] E. H. Putley, Far infra-red photoconductivity, Phys. Status Solidi (b) 6, 571 (1964).

[7] N. Sclar, Properties of doped silicon and germanium infrared detectors, Prog. Quantum Electron. 9, 149 (1984), ISSN 0079-6727.

[8] Hemi H. Gandhi, Hyperdoping Germanium for SWIR Photodetection and High Donor Activation, Degree of Doctor of Philosophy, Harvard University (2019).

[9] Jeffrey M. Warrender, Laser hyperdoping silicon for enhanced infrared optoelectronic properties, Appl. Phys. Rev. 3, 031104 (2016).

[10] Jonathan P. Mailoa, Austin J. Akey, Christie B. Simmons, David Hutchinson, Jay Mathews, Joseph T. Sullivan, Daniel Recht, Mark T. Winkler, James S. Williams, Jeffrey M. Warrender, Peter D. Persans, Michael J. Aziz, and Tonio Buonassisi, Room-temperature sub-band gap optoelectronic response of hyperdoped silicon, Nat. Commun. 5, 3011 (2014), ISSN 2041-1723.

[11] M. J. Aziz, J. Y. Tsao, M. O. Thompson, P. S. Peercy, and C. W. White, Solute Trapping: Comparison of Theory with Experiment, Phys. Rev. Lett. 56, 2489 (1986).

[12] Thomas Gimpel, Stefan Winter, Marcel Boßmeyer, and Wolfgang Schade, Quantum efficiency of femtosecondlaser sulfur hyperdoped silicon solar cells after different annealing regimes, Solar Energy Mater. Solar Cells 180, 168 (2018).

[13] Fang Liu, Mao Wang, Yonder Berencén, Slawomir Prucnal, Martin Engler, René Hübner, Ye Yuan, René Heller, Roman Böttger, Lars Rebohle, Wolfgang Skorupa, Manfred Helm, and Shengqiang Zhou, On the insulator-to-metal transition in titanium-implanted silicon, Sci. Rep. 8, 4164 (2018).

[14] Austin J. Akey, Daniel Recht, James S. Williams, Michael J. Aziz, and Tonio Buonassisi, Single-phase filamentary cellular breakdown via laser-induced solute segregation, Adv. Funct. Mater. 25, 4642 (2015).

[15] Xiaodong Qiu, Zijing Wang, Xiaotong Hou, Xuegong Yu, and Deren Yang, Visible-blind short-wavelength infrared photodetector with high responsivity based on hyperdoped silicon, Photonics Res. 7, 351 (2019).

[16] Mao Wang, A. Debernardi, Y. Berencén, R. Heller, Chi Xu, Ye Yuan, Yufang Xie, R. Böttger, L. Rebohle, W. Skorupa, M. Helm, S. Prucnal, and Shengqiang Zhou, Breaking the Doping Limit in Silicon by Deep Impurities, Phys. Rev. Appl. 11, 054039 (2019).

[17] Philippe K. Chow, Wenjie Yang, Quentin Hudspeth, Shao Qi Lim, Jim S. Williams, and Jeffrey M. Warrender, Observation of enhanced infrared absorption in silicon supersaturated with gold by pulsed laser melting of nanometer-thick gold films, J. Appl. Phys. 123, 133101 (2018).

[18] Meng-Ju Sher, Christie B. Simmons, Jacob J. Krich, Austin J. Akey, Mark T. Winkler, Daniel Recht, Tonio Buonassisi, Michael J. Aziz, and Aaron M. Lindenberg, Picosecond carrier recombination dynamics in chalcogenhyperdoped silicon, Appl. Phys. Lett. 105, 053905 (2014).

[19] W. Yang, N. Ferdous, P. J. Simpson, J. M. Gaudet, Q. Hudspeth, P. K. Chow, J. M. Warrender, A. J. Akey, M. J. Aziz, E. Ertekin, and J. S. Williams, Evidence for vacancy trapping in Au-hyperdoped Si following pulsed laser melting, APL Mater. 7, 101124 (2019).

[20] Naheed Ferdous and Elif Ertekin, Atomic scale origins of sub-band gap optical absorption in gold-hyperdoped silicon, AIP Adv. 8, 055014 (2018).

[21] S. Senali Dissanayake, Philippe K. Chow, Jeffrey M. Warrender, and Meng-Ju Sher, in 2020 45th International Conference on Infrared, Millimeter, and Terahertz Waves (IRMMW-THz) (2020) (to be published).

[22] Tuan T. Tran, Huda S. Alkhaldi, Hemi H. Gandhi, David Pastor, Larissa Q. Huston, Jennifer Wong-Leung, Michael J. Aziz, and J. S. Williams, Suppression of ion-implantation induced porosity in germanium by a silicon dioxide capping layer, Appl. Phys. Lett. 109, 082106 (2016).

[23] Frank A. Hegmann, Oksana Ostroverkhova, and David G. Cooke, in Photophysics of Molecular Materials (John Wiley \& Sons, Ltd, 2005), Chap. 7, p. 367, ISBN 9783527607327.

[24] Haidan Wen and Aaron M. Lindenberg, Coherent terahertz polarization control through manipulation of electron trajectories, Phys. Rev. Lett. 103, 023902 (2009).

[25] M. Mayer, SIMNRA, a simulation program for the analysis of NRA, RBS and ERDA, AIP Conf. Proc. 475, 541 (1999).

[26] P. Hohenberg and W. Kohn, Inhomogeneous electron gas, Phys. Rev. 136, B864 (1964).

[27] W. Kohn and L. J. Sham, Self-consistent equations including exchange and correlation effects, Phys. Rev. 140, A1133 (1965).

[28] G. Kresse and J. Hafner, Ab initio molecular dynamics for liquid metals, Phys. Rev. B 47, 558(R) (1993).

[29] G. Kresse and J. Furthmüller, Efficient iterative schemes for $\mathrm{ab}$ initio total-energy calculations using a plane-wave basis set, Phys. Rev. B 54, 11169 (1996).

[30] Jochen Heyd, Gustavo E. Scuseria, and Matthias Ernzerhof, Hybrid functionals based on a screened coulomb potential, J. Chem. Phys. 118, 8207 (2003).

[31] Aliaksandr V. Krukau, Oleg A. Vydrov, Artur F. Izmaylov, and Gustavo E. Scuseria, Influence of the 
exchange screening parameter on the performance of screened hybrid functionals, J. Chem. Phys. 125, 224106 (2006).

[32] J. P. Perdew, K. Burke, and M. Ernzerhof, Generalized Gradient Approximation Made Simple, Phys. Rev. Lett. 77, 3865 (1996).

[33] John P. Perdew and Wang Yue, Accurate and simple density functional for the electronic exchange energy: Generalized gradient approximation, Phys. Rev. B 33, 8800 (1986).

[34] Kerstin Hummer, Judith Harl, and Georg Kresse, Heydscuseria-ernzerhof hybrid functional for calculating the lattice dynamics of semiconductors, Phys. Rev. B 80, 115205 (2009).

[35] Kuo-Hsing Kao, Anne S. Verhulst, William G. Vandenberghe, Bart Soree, Guido Groeseneken, and Kristin De Meyer, Direct and indirect band-to-band tunneling in germanium-based TFETs, IEEE Trans. Electron Devices 59, 292 (2011).

[36] A. Almazouzi, J. Bernardini, E. G. Moya, H. Bracht, N. A. Stolwijk, and H. Mehrer, Diffusion, solubility, and thermodynamic properties of gold in solid germanium studied by means of radiotracer and spreading-resistance analysis, J. Appl. Phys. 70, 1345 (1991).

[37] Daniel José Silva, Ulrich Wahl, J. G. Correia, L. M. C. Pereira, Lígia Marina Amorim, M. R. Da Silva, E. Bosne, and J. P. Araújo, Lattice location and thermal stability of implanted nickel in silicon studied by on-line emission channeling, J. Appl. Phys. 115, 023504 (2014).

[38] S. B. Zhang and John E. Northrup, Chemical Potential Dependence of Defect Formation Energies in GaAs: Application to Ga Self-Diffusion, Phys. Rev. Lett. 67, 2339 (1991).

[39] M. Gajdoš, K. Hummer, G. Kresse, J. Furthmüller, and F. Bechstedt, Linear optical properties in the projector- augmented wave methodology, Phys. Rev. B 73, 045112 (2006).

[40] John S. Toll, Causality and the dispersion relation: Logical foundations, Phys. Rev. 104, 1760 (1956).

[41] See Supplemental Material at http://link.aps.org/supple mental/10.1103/PhysRevApplied.15.064058 for supporting figures for RBS data, further analysis of carrier recombination rate and sub-band-gap absorption with substitutional $\mathrm{Au}$ dose, defect formation energy change due to change in $\mathrm{Au}$ concentration, and simulated maximum recrystallization velocity.

[42] S. Senali Dissanayake, Matthew Wilkins, Philippe K. Chow, Wenjie Yang, Quentin Hudspeth, Shao Qi Lim, Jim S. Williams, Jeffrey M. Warrender, Jacob J. Krich, and M.-J. Renee Sher, Figure-of-merit evaluation of goldhyperdoped silicon for photovoltaic applications (unpublished).

[43] W. Shockley and W. T. Read, Statistics of the recombinations of holes and electrons, Phys. Rev. 87, 835 (1952).

[44] W. Yang, Q. Hudspeth, P. K. Chow, J. M. Warrender, N. Ferdous, Elif Ertekin, G. Malladi, A. J. Akey, M. J. Aziz, and J. S. Williams, Atomistic Mechanisms for the Thermal Relaxation of Au-Hyperdoped Si, Phys. Rev. Appl. 12, 024015 (2019).

[45] W. Yang, A. J. Akey, L. A. Smillie, J. P. Mailoa, B. C. Johnson, J. C. McCallum, D. Macdonald, T. Buonassisi, M. J. Aziz, and J. S. Williams, Au-rich filamentary behavior and associated subband gap optical absorption in hyperdoped Si, Phys. Rev. Mater. 1, 074602 (2017).

[46] The defect bands are not formed from Au only. Instead, they are strongly hybridized bands of $\mathrm{Au}$ and $\mathrm{Ge}$ with mostly $\mathrm{Ge}$ in it. The Au $d$ level is deep in the valence bands, also hybridized with Ge bulk bands.

[47] Michael F. Millea, The effect of doping on gold diffusion in germanium, J. Phys. Chem. Solids 27, 309 (1966). 